\title{
Effective Chemical Inactivation of Ebola Virus
}

\section{Elaine Haddock, Friederike Feldmann, Heinz Feldmann}

Reliable inactivation of specimens before removal from high-level biocontainment is crucial for safe operation. To evaluate efficacy of methods of chemical inactivation, we compared in vitro and in vivo approaches using Ebola virus as a surrogate pathogen. Consequently, we have established parameters and protocols leading to reliable and effective inactivation.

$\mathrm{T}$ he safe operation of high-level biocontainment laboratories throughout the world is of highest importance. These laboratories are under stringent national oversight and must adhere to international guidelines. Laboratories in the United States that handle select agents are further regulated by the US Centers for Disease Control and Prevention's Division of Select Agents and Toxins and the US Department of Agriculture's Animal and Plant Health Inspection Service.

Proper and reliable inactivation of specimens destined for removal from high-level biocontainment is a critical aspect for laboratory certification and operation. Standard operating procedures (SOPs) are approved by institutional biosafety committees in most cases and additionally by state and/or national regulatory authorities in other cases. In the past, specimens were commonly inactivated on the basis of operational experiences rather than well-documented protocols $(1-3)$.

To evaluate the efficacy of chemical inactivation procedures for specimen removal, we used the US prime select agent and Tier-1 pathogen (4) Zaire ebolavirus (EBOV) as a surrogate model for enveloped high-level containment viruses with single-strand, negative-sense RNA genomes, such as arenaviruses, bunyaviruses, filoviruses, orthomyxoviruses, and paramyxoviruses. These viruses share certain biologic, biochemical, and structural features, making them sensitive to the same chemical inactivation methods. Furthermore, EBOV is currently a prominent example as the causative agent of an unprecedented epidemic in West Africa $(5,6)$.

\section{The Study}

Standard biologic specimens containing infectious EBOV commonly generated in high-level biocontainment

Author affiliation: National Institute of Allergy and Infectious

Diseases, National Institutes of Health, Hamilton, MT, USA

DOI: http://dx.doi.org/10.3201/eid2207.160233 operations were inactivated by several methods of chemical treatment (Figure; Table, http://wwwnc.cdc.gov/EID/ article/22/7/16-0233-T1.htm; online Technical Appendix, http://wwwnc.cdc.gov/EID/article/22/7/16-0233-Techapp1. pdf). For in vitro testing, we used wild-type EBOV expressing enhanced green fluorescent protein (EBOV-eGFP) (7), which allows for cytopathic effect (CPE) and fluorescence as simple readout parameters. For in vivo testing, we used mouse-adapted EBOV (MA-EBOV) (8) infection of BALB/c mice. Virus stocks were grown in Vero E6 cells and titrated by using a $50 \%$ tissue culture infectious dose $\left(\mathrm{TCID}_{50}\right)$ assay (9). Infected cells were produced by infecting Vero E6 cells at a multiplicity of infection of 0.01 . Cells were harvested at CPE of $\approx 75 \%$, pelleted, and resuspended in $6 \mathrm{~mL}$ Dulbecco's phosphate-buffered saline (DPBS); $1 \mathrm{~mL}$ aliquots were stored at $-80^{\circ} \mathrm{C}$. Samples were chemically treated according to the specific testing parameters and dialyzed or run over detergent-removal columns to remove inactivating reagents. In brief, samples were dialyzed by using a 10-kDa molecular weight cutoff (Spectrum Laboratories, Lawrenceville, GA, USA, or Fisher Scientific, Pittsburgh, PA, USA) and using DPBS over a stir plate at $4{ }^{\circ} \mathrm{C}(>500$-fold exchange volumes, 5 changes over 32-48 h); detergent was removed by using DetergentOUT GBS10-5000 columns (G-Biosciences, St. Louis, MO, USA).

Negative control samples included DPBS and noninfected Vero E6 cells and tissue homogenates (mouse); positive control samples included untreated virus stocks and infected Vero E6 cells and mouse tissues. For in vitro testing, all samples were increased in volume to $3 \mathrm{~mL}$ and equally divided to infect Vero E6 cells ( $80 \%$ confluency) in triplicates. Cells were incubated at $37^{\circ} \mathrm{C}$ for 14 days and monitored regularly for CPE or fluorescence. For in vivo testing, samples were increased in volume to $1 \mathrm{~mL}$ and equally divided to infect 5 mice intraperitoneally. BALB/c mice (female, 6-8 weeks old; Charles River Laboratories, Wilmington, MA, USA) were housed in microisolator cages and were monitored daily for 28 days. Because in vitro and in vivo safety testing correlated well, we discontinued mouse infections for ethical reasons.

Nucleic acid extraction is often carried out with commercial guanidinium isothiocyanate buffers. We used Buffer AVL and Buffer RLT (QIAGEN, Valencia, CA, USA) and TRIzol (Life Technologies, Grand Island, NY, USA) according to manufacturers' recommendations. AVL was mixed with stock virus at different ratios, and infected cells were resuspended in RLT (Table). Samples were either 
A
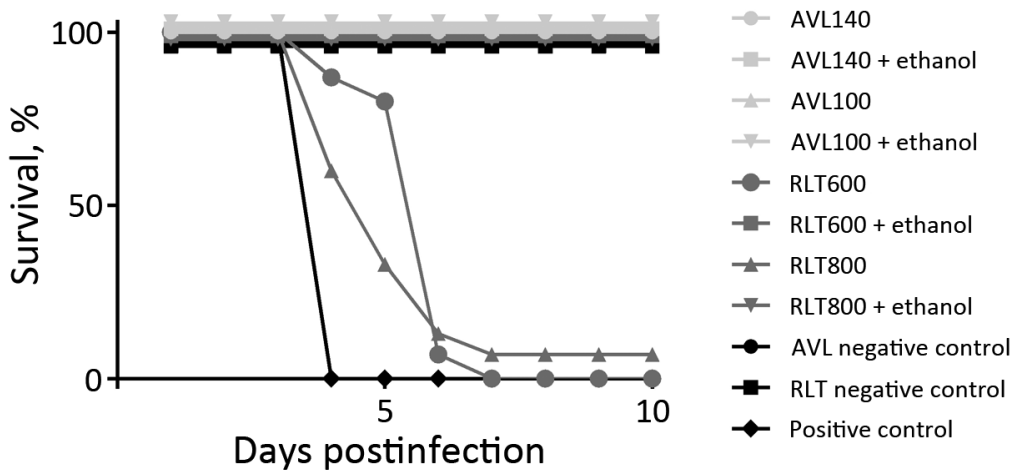

B

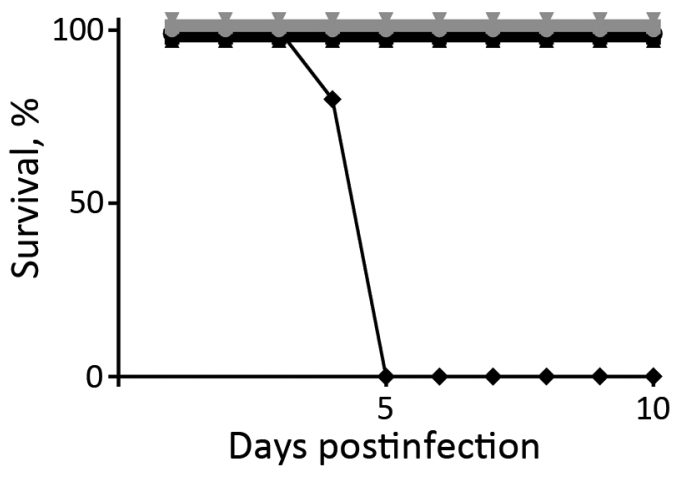

Figure. Ebola virus inactivation results as tested in BALB/c mouse model. A) Survival in animal groups tested with samples inactivated by guanidinium isothiocyanate buffers. AVL140, $140 \mu \mathrm{L}$ Buffer AVL (QIAGEN, Valencia, CA, USA) + 560 $\mu \mathrm{L}$ sample; AVL100, $100 \mu \mathrm{L}$ Buffer $\mathrm{AVL}+600 \mu \mathrm{L}$ sample; RLT600, 600 $\mu \mathrm{L}$ Buffer RLT (QIAGEN) treatment of cells; RLT800, $800 \mu \mathrm{L}$ Buffer RLT treatment of cells; + ethanol, after a Buffer AVL or Buffer RLT inactivation contact time of $10 \mathrm{~min}$, addition of $100 \%$ or $70 \%$ ethanol, respectively, for an additional 20 min of contact time. B) Survival in animal groups tested with samples inactivated by fixative or detergent buffers. For all test groups, $n=15$; for all control groups, $n=5$. immediately dialyzed or treated with ethanol (AVL, 100\% ethanol, $560 \mu \mathrm{L}$; RLT, 70\% ethanol, $600 \mu \mathrm{L}$ ). Infected liver tissue was homogenized in RLT with a stainless steel bead $(10 \mathrm{~min}$ at $30 \mathrm{~Hz})$. A soluble aliquot $(\approx 30 \mathrm{mg})$ was transferred to a new tube, and fresh RLT was added, followed by $70 \%$ ethanol $(600 \mu \mathrm{L})$. After dialysis, samples were used to infect Vero E6 cells and mice. Similar to a results in a previous study (10), AVL and RLT treatment alone for 10 minutes at either ratio did not fully inactivate EBOV; however, the addition of ethanol (the next step of the manufacturer's protocol) rendered all samples completely noninfectious. AVL alone resulted in complete inactivation with longer contact times (i.e., refrigerated overnight or frozen for 7 days) (Table; Figure).

Infected cells were resuspended and treated with TRIzol (1:4 vol/vol). Infected liver samples were homogenized in $1 \mathrm{~mL}$ TRIzol as described in the previous paragraph. After centrifugation, an aliquot of tissue homogenate $(\approx 50 \mathrm{mg})$ was transferred to a new tube, and fresh TRIzol was added. Additionally, blood from infected animals was mixed ( $1: 4 \mathrm{vol} / \mathrm{vol})$ with TRIzol. After dialysis, Vero E6 cells were inoculated and monitored for CPE or fluorescence. In all cases, virus growth was not detected (Table), indicating complete inactivation.
Formalin, paraformaldehyde, and glutaraldehyde can be used to fix cells or tissues for histologic or microscopic studies. Infected cells were diluted $1: 4$ in $10 \%$ neutralbuffered formalin $(7.5 \%$ fixative) or $1: 5$ in either $2.5 \%$ glutaraldehyde or $2.5 \%$ paraformaldehyde (2\% fixative). Samples were dialyzed and used to infect Vero E6 cells or mice. Monitoring of cell culture and animals resulted in the absence of CPE or fluorescence and clinical signs, respectively, indicating complete inactivation of EBOV (Table; Figure).

Infected liver segments were incubated in $10 \%$ neutral-buffered formalin, $2 \%$ glutaraldehyde, or $2 \%$ paraformaldehyde $(10 \mathrm{~mL})$ for a period of 7 days $\left(<1-\mathrm{cm}^{3}\right.$ piece) or 30 days $\left(>1-\mathrm{cm}^{3}\right.$ piece) at $4^{\circ} \mathrm{C}$. Subsequently, a small section of tissue $(\approx 150 \mathrm{mg})$ was dissected, homogenized in DPBS with a stainless steel bead (10 min at $30 \mathrm{~Hz}$ ), and then dialyzed. After dialysis, samples were used to infect Vero E6 cells. All samples were completely inactivated (Table).

Samples for protein assays are often inactivated by a combination of detergent and heat. We tested the parameters of $60^{\circ} \mathrm{C}$ for $30 \mathrm{~min}, 65^{\circ} \mathrm{C}$ for 15 or $30 \mathrm{~min}$, and $70^{\circ} \mathrm{C}$ for $15 \mathrm{~min}$ in conjunction with a buffer containing $0.5 \%$ Triton X-100 and 0.5\% Tween-20 (both from Sigma-Aldrich, 
St. Louis, MO, USA); this mixture is commonly used for ELISA. Stock virus was diluted 1:25 in this buffer and heated for the appropriate times before samples were clarified of detergent and used to infect Vero E6 cells or mice. All samples were completely inactivated as indicated by lack of CPE or fluorescence in cells and clinical signs in mice (Table; Figure).

Boiling (at $100^{\circ} \mathrm{C}$ for $10 \mathrm{~min}$ or $120^{\circ} \mathrm{C}$ for $5 \mathrm{~min}$ ) might be sufficient to inactivate EBOV (Table) (11) but is often used in conjunction with sodium dodecyl sulfate (SDS)containing buffers for protein analysis. Aliquots of infected cells were diluted in DPBS and $4 \times$ loading buffer $(1 \%$ SDS final). Infected liver tissue $(\approx 150 \mathrm{mg})$ were placed in DPBS and $4 \times$ loading buffer ( $1 \%$ SDS final). The samples were then homogenized with a stainless steel bead (10 min at 30 $\mathrm{Hz}$ ). After detergent removal, samples were used to infect Vero E6 cells; all treated cells and tissue homogenates were negative for infectious EBOV (Table).

\section{Conclusions}

Our study establishes inactivation procedures for EBOV that can be safely applied to distinct specimen types and research purposes and might also apply to other enveloped, single-strand, negative-sense RNA viruses. Our findings should help to improve and approve SOPs for inactivation without the need for safety testing each individual sample, an unfeasible and unwarranted task in current diagnostic and research operations in high-level biocontainment settings. However, any changes to inactivation SOPs make further safety testing essential. Safety testing for inactivation, at least for EBOV, can rely on cell culture only because this seems to be as sensitive as in vivo testing.

\section{Acknowledgments}

The authors are grateful to the Rocky Mountain Veterinary Branch of the National Institute of Allergy and Infectious Diseases (NIAID) at the National Institutes of Health (NIH) for assistance with animal work.

The study was supported by the Intramural Research Program of NIAID at NIH.
Dr. Haddock is a biologist in the Laboratory of Virology, Division of Intramural Research, NIAID, NIH. Her interests include high-level biocontainment operations and emerging zoonotic pathogens.

\section{References}

1. Mitchell SW, McCormick JB. Physicochemical inactivation of Lassa, Ebola, and Marburg viruses and effect on clinical laboratory analyses. J Clin Microbiol. 1984;20:486-9.

2. Elliott LH, McCormick JB, Johnson KM. Inactivation of Lassa, Marburg, and Ebola viruses by gamma irradiation. J Clin Microbiol. 1982;16:704-8.

3. Lupton HW. Inactivation of Ebola virus with 60Co irradiation. J Infect Dis. 1981;143:291. http://dx.doi.org/10.1093/ infdis/143.2.291

4. US Centers for Disease Control and Prevention, US Department of Agriculture. Select agents and toxins list [cited 2016 Mar 29]. http://www.selectagents.gov/SelectAgentsandToxinsList.html

5. World Health Organization. Ebola virus disease outbreak: Ebola is no longer a public health emergency of international concern [cited 2016 Mar 29]. http://www.who.int/csr/disease/ebola/en

6. US Centers for Disease Control and Prevention. 2014 Ebola outbreak in West Africa [cited 2016 Mar 29]. http://www.cdc.gov/ vhf/ebola/outbreaks/2014-west-africa/index.html

7. Ebihara H, Theriault S, Neumann G, Alimonti JB, Geisbert JB, Hensley LE, et al. In vitro and in vivo characterization of recombinant Ebola viruses expressing enhanced green fluorescent protein. J Infect Dis. 2007;196(Suppl 2):S313-22. http://dx.doi.org/10.1086/520590

8. Bray M, Davis K, Geisbert T, Schmaljohn C, Huggins J. A mouse model for evaluation of prophylaxis and therapy of Ebola hemorrhagic fever. J Infect Dis. 1998;178:651-61. http://dx.doi.org/ $10.1086 / 515386$

9. Marzi A, Feldmann F, Hanley PW, Scott DP, Günther S, Feldmann H. Delayed disease progression in Cynomolgus macaques infected with Ebola virus Makona strain. Emerg Infect Dis. 2015;21:1777-83. http://dx.doi.org/10.3201/eid2110.150259

10. Smither SJ, Weller SA, Phelps A, Eastaugh L, Ngugi S, O'Brien LM, et al. Buffer AVL alone does not inactivate Ebola virus in a representative clinical sample type. J Clin Microbiol. 2015;53:3148-54. http://dx.doi.org/10.1128/JCM.01449-15

11. Chong YK, Ng WY, Chen SP, Mak CM. Effects of a plasma heating procedure for inactivating Ebola virus on common chemical pathology tests. Hong Kong Med J. 2015;21:201-7. http://dx.doi.org/10.12809/hkmj144373

Address for correspondence: Heinz Feldmann, Rocky Mountain Laboratories, 903 S 4th St, Hamilton, MT 59840, USA; email: feldmannh@niaid.nih.gov 